\title{
$\underline{\text { Forensic Mental Health Treatment and Recidivism }}$
}

Daniel Whiting, Howard Ryland and Seena Fazel

This is the submitted version of a chapter now available in:

Focquaert F, Shaw E \& Waller BN (Eds). The Routledge Handbook of the Philosophy and Science of Punishment. $1^{\text {st }}$ Edition. Routledge, 2021. DOI:

https://doi.org/10.4324/9780429507212

\section{$\underline{\text { Introduction }}$}

Forensic mental health services are provided typically for people with severe mental illnesses who have committed violent offences, and tend to refer to secure hospitals which treat and manage individuals who are transferred from court or prison. In the UK, these services are described by those commissioning them as being for 'individuals who have a mental disorder and pose or have posed risks to others'(JCPMH, 2013). There is typically a link between the mental disorder and that risk.

Such individuals are commonly located in hospitals, usually specialised secure units, but also in prisons or in the community under supervision. Forensic mental health services work collaboratively with a wide range of other health and justice professionals and services (Eastman et al., 2012a).

\section{$\underline{\text { The International Context }}$}

There are significant variations worldwide in the availability, structure and function of forensic mental health services. This is a result of a complex interplay between a wide range 
of factors, including funding, the legal context and the structure of healthcare services (Nedopil, 2009, Abdalla-Filho and Bertolote, 2006, Arboleda-Florez, 2006).

Firstly, the total level of funding available for all mental health services varies significantly between countries. According to the World Health Organisation, the global median expenditure on mental health is 2.5 USD. In many lower income countries, mental health services receive a smaller proportion of the overall health budget, which can result in very low levels of funding assigned to mental health services. Consequently, the services provided in lower income countries may be limited in nature, with little provision for more specialised services, such as forensic mental health. Most mentally ill individuals in such countries who commit offences are likely to be dealt with by the criminal justice system. Forensic hospital services that do exist may in practice be extensions of prisons (Njenga, 2006). At the same time, in resource-poor settings, mentally disordered individuals who behave in a violent manner may be more likely to come to the attention of mental health services, due to the disruptive nature of their behaviour. In higher income countries, there may be substantial resources ring-fenced for forensic mental health services (Wilson et al., 2011). The European region spends 20 times as much per capita on mental health than Africa or South East Asia (WHO, 2018). This can facilitate the provision of a sophisticated range of specialised services, specifically for mentally disordered persons who have committed serious offences (Arboleda-Florez, 2006).

Secondly, criminal law varies significantly between jurisdictions, with consequences for the provision of forensic mental health services (Edworthy et al., 2016). Most countries worldwide now have a specific mental health act, with 111 countries reporting that they have stand-alone mental health legislation in 2017, which represents $57 \%$ of World Health 
Organization member countries (WHO, 2018). Despite these advances, a sizeable minority of countries still do not have such legislation, while in other jurisdictions the legislation may be outdated (Zhang et al., 2015). Only certain countries have dedicated provision within their legal framework that governs the judicial management and medical treatment of mentally ill individuals, who come into contact with the criminal justice system. Frameworks vary in a number of respects, such as whether prisoners can be involuntarily given psychiatric medications, which has an impact on the way forensic mental health services are organised (Eastman et al., 2012b). There is also considerable variation in the public perception of mentally ill individuals who offend, which relates to the perceived degree of culpability for crimes committed when unwell. This can have an impact on the legal outcome from the courts for mentally ill individuals and transitions between the criminal justice and mental health systems (Meynen and Oei, 2011). Due to the lengthy process required to create and modify statute law in many jurisdictions, the legal situation can often lag behind contemporary practical and ethical norms (Simpson, 2015).

Finally, the structure and funding of the healthcare and criminal justice system shows considerable variation between countries and even within countries. This can have a sizeable impact on the way that services are provided for mentally ill individuals who pose a risk to others. In some countries, forensic mental health services may be exclusively provided and paid for by the state, while in others a range of providers may be allowed to compete for business, which may be whole or partly commissioned by the state (Abdalla-Filho and Bertolote, 2006).

Given the complex differences between forensic mental health services worldwide, it is not possible to provide a comprehensive description of all such services in this chapter. We will 
therefore use the example of how services are provided in England. This provides an illustration of how such services can be organised within a high-income setting, and English services have acted as a model for service development in other countries.

\section{Overall Structure of Forensic Mental Health Services in England}

Forensic mental health services in England are highly developed and receive around $£ 1.3$ billion funding each year, which is equivalent to around $19 \%$ of the entire national mental health budget (Wilson et al., 2011).

The backbone of these services are the secure hospitals, which provide inpatient care within the healthcare system. Increasingly many regions now have some form of specialised forensic community mental health team to manage individuals discharged from secure hospitals. Within the criminal justice system, there is also increasing provision of liaison services (between the courts and community mental health teams) for individuals with mental illness. In addition, prisons will usually have some form of mental health provision. There are also an expanding range of services that provide liaison with earlier stages of the criminal justice system, including police stations and criminal courts (Durcan et al., 2011).

\section{$\underline{\text { Secure Hospitals }}$}

England has a long history of providing inpatient forensic mental health care, starting in 1863 with the establishment of the Broadmoor Criminal Lunatic Asylum. This was established as a humane alternative to the prison system for the 'criminally insane', which could provide some measure of treatment, as well as containment (Stevens, 2013). Over the subsequent 
century other 'special hospitals' were also opened to manage increased numbers of individuals. Reforms initiated in the 1960s led to the creation of lower tiers of security and further expansion of capacity from the 1970s onwards (Seppänen et al., 2018).

Currently there are around 6000 inpatient beds available in secure hospital services across England (JCPMH, 2013). These are arranged over three levels of security, namely high, medium and low, with the majority of individuals located in the lower two. Services are commissioned nationally as part of specialised commissioning at NHS England, which receives funding directly from the Department of Health. The delivery of services is performed by a range of providers, including the National Health Service, charitable hospital organizations and private companies (Hare Duke et al., 2018).

There are currently three high secure hospitals in England, namely Broadmoor, Rampton and Ashworth Hospitals. These cater for the highest risk individuals who pose a serious and immediate danger to the public. Security measures resemble those at a prison, with high walls, airport style screening and high staffing levels. Medium and low security hospitals offer progressively lower levels of security, but usually still require that all patients are detained under the Mental Health Act (Durcan et al., 2011). These have security in the form of physical, procedural and relational measures, such as perimeter fencing, searching of patients returning from leave and the development of a therapeutic relationship between patients and a named member of staff (Chester et al., 2017).

Patient admissions are usually lengthy, typically lasting 2-10 years (Hare Duke et al., 2018). A recent study found that $20 \%$ of patients in high and medium security stayed for more than 20 years (Völlm et al., 2018). Individuals will enter the system from different routes. Many 
will have treatment mandated by a court as an alternative to a prison sentence. Others will be transferred from prison, either before or after conviction. Less commonly some patients will be transferred from general psychiatric services, due to unmanageable levels of aggression.

Treatment within secure services is multidisciplinary and includes optimisation of pharmacological management, psychotherapeutic interventions, occupational therapy and a range of other therapeutic activities. Patients will usually receive progressively more leave from the hospital. Initially they will be escorted by staff and if this is judged to be successful, will be given unescorted leave, in preparation for discharge (Eastman et al., 2012c).

\section{Community Forensic Mental Health Teams}

Complementing these inpatient services, there are clinical teams that provide specialised services to patients who have been discharged from secure care. These are currently varied in their structure, function and funding, although there are moves to create greater standardisation in the model of provision. Responsibility for care may be shared with adult community services. The majority of patients will be discharged in a highly structured way, so that they are required to live in some form of supported accommodation, with regular review by mental health and other professionals, with provisions for returning them to hospital if concerns are raised about increasing risk or deteriorating mental health (Natarajan et al., 2018).

\section{$\underline{\text { Prison Mental Health Services }}$}


Rates of mental illness in prison are high, with around 1 in 7 with a psychotic illness or major depressive disorder (Fazel et al., 2016a). A recent survey in England and Wales found that almost all prisons will have some specialised mental healthcare provision, although $13 \%$ of prisons had no such services (Forrester et al., 2013). The study concluded that services can be highly variable in their structure and the level of support offered. Some prisons will have a dedicated healthcare area, where prisoners with advanced needs can be more closely monitored and treated. The majority of mental illness is treated within the prison context, however in England, the Mental Health Act does not sanction the use of involuntary medication. If a prisoner becomes mentally unwell and refuses treatment, then they can be referred for a transfer to a hospital, under the Mental Health Act (Bradshaw et al., 2017).

\section{Liaison and Diversion Services}

A relatively recent addition to national forensic mental health services are mental health professionals, usually nurses, who provide brief assessments at police stations and courts. These liaison and diversion services were a policy priority arising from the Bradley review, a government-sponsored inquiry to examine the extent to which offenders with mental health problems, or learning disabilities, could be diverted to other services and the barriers to such diversion. The resultant recommendations emphasised prevention and early intervention (Bradley, 2009). Liaison services in police stations are used to screen people following arrest who are known to have, or appear to be suffering from, mental illness. Additionally, police officers have the power under the Mental Health Act to take people to a place of safety for assessment by mental health professionals. 
Liaison services in courts are usually focussed on Magistrates' Courts, which are the lower criminal courts in England, and deal with less serious offences. Referrals to such teams can come from prisons, custody officers, probation officers or a range of other sources. Liaison services aim to assist the criminal justice process, advise courts on appropriate disposal, signpost individuals to relevant services and divert those requiring assessment or treatment to the relevant healthcare services (Birmingham et al., 2017).

A recent evaluation of 10 pilot sites implementing the new national service model concluded that such services led to better identification of individuals with vulnerabilities and an improved provision of timely, relevant information to the police, courts and partner organizations. There were statistically significant increases in the average number of cases for which appointments were offered for learning disability, which increased from 0.39 to $1.22(\mathrm{p}=0.001)$ and for financial appointments, which increased from 0.12 to $0.92(\mathrm{p}=0.012)$. The authors identify limitations to the data available for analysis and conclude that the qualitative information collected from stakeholders is the main strength of the evaluation (Disley et al., 2016).

\section{$\underline{\text { Recidivism in Forensic Mental Health Services }}$}

One of the main aims of forensic mental health services is to reduce reoffending in those discharged from secure care. Other important outcomes are preventing early mortality, especially from suicide, and the need for rehospitalisation.

A systematic review and meta-analysis examined patient outcomes following discharge from secure hospitals and specifically reported on reoffending, all-cause mortality, suicide and 
readmission (Fazel et al., 2016a). This review identified 35 papers that met the inclusion criteria, of which 30 studies provided information about reoffending. The crude reoffending rate for forensic patients ranged from 0 to 24,244 per 100,000 person-years. The pooled estimate was 4484 per 100,000 person-years (with a 95\% confidence interval of 3679-5287). The review also compared this rate with other potentially comparable groups, such as released prisoners of similar age, and individuals who have committed crimes with personality disorder or mental illness. In these studies, the rates of reoffending varied from 4,535 to 36,964 per 100,000 person-years. The group with the highest rate of reoffending was prisoners who were released on probation. Fifteen studies identified specifically reported violent reoffending for patients in forensic mental health services, which was a pooled rate of 3,902 per 100,000. Prevalence ratios were calculated comparing reoffending in forensic patients with prisoners, matched on factors such as country and age. In the UK, the prevalence ratios ranged from 1.4 to 7.7 and in the USA from 2.7 to 5.0.

This review concluded that, based on the information available in the literature, rates of reoffending appeared to be lower in forensic patients than those in comparable groups such as prisoners. However, there are a number of challenges in comparing these two populations, especially as forensic patients tend to have committed more serious offences and have a longer time in hospital. Several possible explanations may help to account for the observed differences (Fazel et al., 2016a). Patients accepted for inpatient forensic psychiatric treatment are usually carefully selected, often on the basis of perceived treatability, which contrasts to the unfiltered prison population. Forensic patients also differ in the profile of crimes committed, with higher rates of more serious, violent crimes than the equivalent prison populations. Perpetrators of acquisitive crimes, drug offences and minor violent crimes are more likely to reoffend, and these make up the majority of prisoners. In addition, forensic 
patients may have committed their offence when acutely unwell, which is associated with a low risk of reoffending, especially if receiving regular follow up and ongoing treatment in the community.

\section{Assessing Risk of Recidivism}

A period of admission within a forensic mental health service has a range of goals. These include optimising a patient's mental and physical wellbeing, and improving function across broad social and occupational domains. A fundamental task however is developing a clear understanding of an individual's risks of reoffending. This in part guides treatment decisions, and ultimately the timing and nature of reintegration into community settings. Indeed, it is an expectation of any Court that if someone has been convicted and transferred to secure hospital, this is because such a pathway is the most suitable to address future risk. In the main part, it is the risk of future violent offending that is of concern, and the majority of secure psychiatric patients have a history of violent offending (Fazel et al., 2016b).

Risk assessment is a dynamic process that is repeated and updated throughout an individual's contact with forensic mental health services. It is a core part of progress reviews and planning meetings. All members of the multi-disciplinary team of clinicians may contribute. Typically this team will include doctors, nurses, psychologists, occupational therapists and social workers, each of whom may provide information regarding different relevant factors. Whilst approaches vary, all assessments to some extent combine different factors and observations associated with future offending to formulate an overall estimation of risk. Such assessments may also seek to predict the nature that any offending may take, and the circumstances in which it may occur. Factors considered may be 'static' or historical, such as a past offence, or 'dynamic', changing over time and potentially modifiable such as substance misuse. 
To guide risk assessments, clinicians in secure hospital settings often utilise structured assessment tools (Khiroya et al., 2009). In antisocial personality disorder, the use of such a tool is recommended in guidelines produced by the National Institute for Health and Care Excellence in England (NICE, 2009), and their use is also a performance indicator for forensic units in some countries, such as England. Tools generally involve either an actuarial approach of using weighted or unweighted factors to generate a probabilistic estimate of future violence, or 'structured clinical judgement' in which clinicians use the presence or absence of certain factors as a basis for their risk stratification. The latter approach is more common in secure hospital settings, as it can potentially guide treatment planning. However, the accuracy of currently used structured instruments for such management decisions has been questioned (Coid et al., 2015a), and other limitations include requiring significant time, money and training (Singh et al., 2011), and the presence of authorship bias in validation studies (Singh et al., 2013). Whilst these tools can more accurately assign low-risk status, their positive predictive ability is not such that they should be relied on in isolation for decisions around sentencing or discharge (Fazel et al., 2012).

\section{Factors Associated with Recidivism}

A Swedish longitudinal study examined the rate of conviction for violent offences for a cohort of 2248 patients discharged from secure psychiatric hospitals into community settings between 1992 and 2013 (Wolf et al., 2018). Linking of several national data registers provided information on risk factors, and violent conviction data was collected for up to 24months following discharge. The strongest predictors of violent offending were previous violent crime (hazard ratio 3.2, 95\% confidence interval 2.3 to 4.5 ) and sex (female vs. male hazard ratio $0.4,95 \%$ confidence interval 0.3 to 0.6 ). Lifetime drug use disorder was also 
associated with future violent offending (hazard ratio $2.2,95 \%$ confidence interval 1.7 to 2.9). The importance of substance use disorder comorbidity for risk of violent offending is also clearly demonstrated in broader psychiatric populations (Fazel et al., 2009), and recent substance use has been identified as a dynamic risk factor for perpetration of a violent offence (Witt et al., 2013). Assessing an individual's substance use and its relevance to past offending is therefore a key part of addressing risk in secure settings.

This Swedish study also found a length of inpatient stay of 12-months or more to be associated with a lower risk of violent offending on release, as was a history of five or more previous inpatient episodes (Wolf et al., 2018). This may be related to post-discharge supervision, and the extent to which services are able to develop knowledge of a patient over time. When compared with no history of violent crime, a previous history of serious violent crime (defined as homicide, aggravated assault, aggravated robbery, rape, sexual coercion or sexual exploitation) was associated with a lower increased risk of recidivism than history of any violent crime. Low rates of recidivism for grave crimes such as homicide when associated with mental illness have elsewhere been demonstrated (Golenkov et al., 2014). This may reflect the effectiveness of long term treatment and supervision for such individuals in areas where forensic psychiatric services are established.

Psychiatric diagnosis is also associated with recidivism. Rates of post-discharge violent offending in different diagnostic groups were examined in a cohort of 6,505 patients discharged from forensic psychiatric hospitals in Sweden (Fazel et al., 2016b). Over a mean follow-up of 9.4 years, a primary diagnosis of personality disorder (characterised by pervasive and impairing patterns of behaviour and modes of relating to oneself and others) was associated with the highest rates of violent offending (hazard ratio relative to reference 
category of schizophrenia-spectrum disorders $1.73,95 \%$ confidence interval 1.57 to 1.92 ). Relative to schizophrenia-spectrum disorders, substance use disorder and bipolar disorder were also associated with increased hazards of post-discharge violent offending, but unipolar depression was not. Personality disorder was also associated with the highest rates of reoffending in 1344 patients who were discharged from medium secure psychiatric settings in England and Wales and followed up for an average of six years (Coid et al., 2015b).

In clinical practice, judgements around recidivism risk are also informed by more qualitative observations of a patient's clinical course, therapeutic engagement, interpersonal functioning and patterns of behaviour. Whilst such parameters have been less robustly epidemiologically linked to future violent offending, few clinicians would omit such complementary knowledge from their assessments. Developing this in-depth individualised knowledge assists in understanding and managing risk both in the short to medium term on a secure unit, and in the longer term in supervised community settings. Of these types of observation, factors including lack of insight or noncompliance, impulsivity, negative attitudes and hostility are among the more strongly associated with recidivism in released male prisoners (Coid et al., 2011) and with violence outcomes in a meta-analysis of 45,533 individuals with psychotic illness (Witt et al., 2013).

\section{$\underline{\text { Treatment in Forensic Mental Health Care }}$}

Where there is a link between offending and mental illness, forensic services assume that evidence-based treatment of the underlying mental disorder will result in a reduction of criminal behaviour. Such treatment is usually initiated in hospital and may include a number of components (Howner et al., 2018). In practice, typically in the first months of a hospital admission, the presence of mental illness in the acute phase requires a largely medication- 
based approach, before entering a more multi-faceted rehabilitation phase of treatment with the longer term goals of maintaining stability and reducing recidivism.

\section{Medication}

Almost all patients in forensic mental health services will receive some form of long term psychopharmacotherapy. Evidence in support of pharmacotherapy to reduce violent behaviour comes from both randomised controlled trials (Leucht et al., 2012) and observational studies (Chang et al., 2016, Fazel et al., 2014). An inpatient admission provides an intensively supported healthcare environment, and an opportunity to either commence a trial of medication, restart previously effective medication, or optimise a patient's medication regimen. This includes identifying which medication or combination of medications is most effective at controlling symptoms of mental illness. In some cases, the nature of an individual's presentation may necessitate coercive use of medication under the framework of relevant mental health law as a last resort, when a collaborative approach has failed. The side effect profile of medications is important, ensuring that these are tolerable for the individual and weighing longer terms potential harms against benefits. The route of administration is also often relevant, for example considering a long-acting depot injection for antipsychotic medication, which can be more easily monitored and can ensure improved adherence. The main challenges arise following discharge, where much lower levels of supervision may mean that patients stop taking their medications, resulting in relapse and potentially an increased risk of aggression.

In terms of classes of medication, antipsychotic medication was shown to reduce violent crime by $45 \%$ in a within-individual observational study of 82,647 individuals in Sweden, and a reduction was also seen of $24 \%$ with mood-stabilising medication (Fazel et al., 2014). 
When separated by diagnosis, mood-stabilising medication was associated with a reduction in violent offending only in those with a diagnosis of bipolar disorder. These data also demonstrated the association of depot formulations with reduced offending. It did not however support the addition of mood stabilisers to antipsychotic medication to reduce offending, an important finding that should support initiatives to reduce the use of polypharmacy for this purpose. A particular antipsychotic medication, clozapine, used in treatment-resistant schizophrenia, was associated with reductions in arrest rates (although the investigation was underpowered to test its association with conviction). Other work suggests that clozapine may have superior anti-aggressive effects (Topiwala and Fazel, 2011), that are not explained by better psychotic symptom control or sedation alone (Frogley et al., 2012).

Medication to treat attention deficit hyperactivity disorder (ADHD) has also been shown to reduce offending when time periods for an individual being on- and off-medication were compared for 25,656 individuals with ADHD (Lichtenstein et al., 2012), and also in an observational study of released prisoners (Chang et al., 2016). Whilst a disorder like ADHD would not be a primary reason for admission into forensic mental health services, identification and treatment of such important comorbidities are a potential advantage of a more intensive assessment that can be provided in forensic psychiatry.

\section{Substance Use}

Substance misuse is common in people under the care of forensic mental health services and has been shown to significantly increase a number of adverse outcomes, including repeat offending. Treatment of substance misuse is therefore a priority for those affected, and many secure units will provide programmes targeting it. Mandatory drug testing may form part of both the procedural security of a unit and supervised community follow-up after discharge. Opioid substitution medications, methadone and buprenorphine, were shown to specifically 
reduce arrests for violent crime in a total population cohort study (Molero et al., 2018), and observational data has also shown pharmacological treatment of substance use disorders to be associated with substantially lower rates of violent reoffending in individuals with comorbid schizophrenia spectrum disorders or bipolar disorder (Chang et al., 2016).

\section{Psychological Therapies}

Most patients will be expected to engage with psychotherapeutic interventions in preparation for release. The emphasis placed on psychological intervention varies between patients, often depending on diagnosis. The purpose of therapy can be wide ranging, but can focus on management of anger, increasing tolerance of frustration, gaining insight into the index offence and triggers for a relapse of symptoms. Patients are often encouraged to make some form of relapse prevention plan, and clinicians may use an individual's ability to engage in such a piece of work as a guide to readiness for less restrictive settings. Psychotherapy broadly aims to equip patients with longer term tools to sustain general well-being and avoid interpersonal conflicts.

Individuals with a primary diagnosis of personality disorder may receive a form of psychotherapy as their primary treatment. In England, national guidelines for the treatment of individuals with antisocial personality disorder and a history of offending recommend offering group-based cognitive and behavioural interventions with a focus on reducing offending and other antisocial behaviour (NICE, 2009). Impulsivity and interpersonal difficulties may be targets for treatment. Overall however, good quality evidence for the efficacy of particular psychological interventions to reduce aggression or reoffending in antisocial personality disorder is somewhat limited (Gibbon et al., 2010), and is a research priority going forward. 


\section{Occupational Therapy}

Many admitted to secure services lack basic skills in self-management. This may be because they have either never learned such skills, or these have been lost due to prolonged illness or institutionalisation. Admission aims to maximise functioning and independence. The ability to structure time appropriately is fundamental for long term rehabilitation. Occupational therapy is considered important to achieving this and focuses on supporting the development of essential practical skills and fostering creativity. Although the specific contribution of occupational therapy to reducing recidivism has yet to be empirically demonstrated, it is undertaken as part of a multidisciplinary approach to improve wellbeing and reduce risk. Themes of volitional realignment towards prosocial goals and experiences, increasing protective factors and community integration have been identified as relevant to this goal (Connell, 2016).

Other factors that are important in ensuring patients have stability on discharge include the identification of suitably supportive accommodation, links with primary medical services, and the support of family.

\section{Pooled Evidence for Interventions}

A recent umbrella review demonstrated that in both general and forensic psychiatric settings, high-level evidence for targeted interventions to reduce the risk of violent offending is limited (Wolf et al., 2017). Ten databases were searched for systematic reviews of violence prevention interventions within general psychiatry, forensic psychiatry, and individuals with mental disorders who had offended. Umbrella reviews such as this incorporate existing systematic reviews and meta-analyses to allow comparison of multiple interventions, and identify areas deficient in evidence. Three systematic reviews and two meta-analyses including a total of 8876 patients were identified. However, none specifically included 
forensic psychiatric populations. Any conclusions drawn will therefore be extrapolated from evidence in other populations.

The strongest evidence was a meta-analysis of moderate quality that examined interventions to prevent relapse into criminality, drug use or both for individuals in the justice system with mental disorder and comorbid substance use (Perry et al., 2015). This meta-analysis was based on 8 included trials with diverse interventions in the United States justice system, from case management to interpersonal psychotherapy. The only positive evidence was for therapeutic community treatment - long-term, structured daily activity programmes with a group-based approach used widely in the treatment of substance use disorders. Of three randomised studies that examined this type of intervention $(n=636)$, two found a significant reduction in re-incarceration. One was a 12-month programme for prisoners of psychoeducational classes, cognitive-behavioural methods, medication and group therapy followed by aftercare of counselling, medication, psychiatric services and basic skills (relative risk [RR] 0.3, 95\% CI 0.1-0.6). The second examined a 6-month residential programme undertaken alongside working in the community, in which participants had incremental responsibility in the group and attended various psychoeducational classes and counselling (RR $0.5,95 \%$ CI $0.3-0.9$ ). A third randomised study of a therapeutic community programme found no superiority to cognitive-behavioural intervention. Another review also found that a modified therapeutic community approach that focused on treating substance abuse, mental illness and criminal thinking and behaviour reduced re-incarceration in individuals with antisocial personality disorder (Wilson, 2014).

Lower quality evidence was also found for other approaches to individuals with mental disorder within the criminal justice system. One meta-analysis found an overall effect on 
violent crime for 6 different interventions $(n=7,677)$, with a pooled effect size of $0.2(95 \%$ confidence interval 0.03-0.5) (Martin et al., 2012). The interventions were correctional programmes, psychiatric treatment, use of a mental health court, forensic psychiatric aftercare, a maximum security therapeutic community for individuals with psychopathy, and intensive community treatment. The effects were not broken down by individual intervention, so it is not possible to identify the therapeutic ingredients with the clearest effects.

Individual trials have shown the potential for interventions more widely evaluated in forensic groups without mental illness to translate to forensic psychiatric populations. A randomised controlled trial in medium secure units in the UK examined the effectiveness of a cognitive skills programme targeting problem-solving skills and thinking styles (Cullen et al., 2012). Individuals who completed the programme showed a reduction in inpatient violence during treatment, though only effects on verbal aggression and substance use were maintained at 12month follow-up. There is a need for evaluation and development of such targeted treatments. The process alone of structured risk assessment to guide shared treatment-planning between clinician and patient has been shown to be insufficient to reduce recidivism in a cluster randomised controlled trial including 632 forensic psychiatric outpatients (Troquete et al., 2013).

\section{Summary}

Forensic mental health services have been developed to meet the diverse needs of individuals with mental illness within the criminal justice system, and to provide a specialist approach to assessing and managing the risk of reoffending in this population. Such provision typically involves significant resource and a multi-disciplinary team of mental health clinicians working in secure hospitals, prisons and the community. There is a need for high-quality 
treatment research in forensic psychiatric populations, ideally using standardised and objective measures of recidivism. However, emerging research suggests that complex interventions targeted at mental health needs, substance abuse, and criminal behaviour can reduce recidivism even in individuals with comorbidities.

ABDALLA-FILHO, E. \& BERTOLOTE, J. M. 2006. Forensic psychiatric systems in the world. Revista Brasileira de Psiquiatria, 28, 56-61.

ARBOLEDA-FLOREZ, J. 2006. Forensic psychiatry: contemporary scope, challenges and controversies. World Psychiatry, 5, 87-91.

BIRMINGHAM, L., AWONOGUN, L. \& RYLAND, H. 2017. Diversion from custody: An update. BJPsych Advances, 23, 375-384.

BRADLEY 2009. Lord Bradley's Review of People with Mental Health Problems or Learning Disabilities in the Criminal Justice System. London, UK: House of Lords.

BRADSHAW, R., PORDES, B. A., TRIPPIER, H., KOSKY, N., PILLING, S. \& O'BRIEN, F. 2017. The health of prisoners: summary of NICE guidance. British Medical Journal, 356, j1378.

CHANG, Z., LICHTENSTEIN, P., LÅNGSTRÖM, N., LARSSON, H. \& FAZEL, S. 2016. Association between prescription of major psychotropic medications and violent reoffending after prison release. Journal of the American Medical Association, 316, 1798-1807.

CHESTER, V., ALEXANDER, R. T. \& MORGAN, W. 2017. Measuring relational security in forensic mental health services. Psychiatric Bulletin, 41, 358-363.

COID, J. W., KALLIS, C., DOYLE, M., SHAW, J. \& ULLRICH, S. 2015a. Identifying causal risk factors for violence among discharged patients. PLOS ONE, 10, e0142493.

COID, J. W., YANG, M., ULLRICH, S., HICKEY, N., KAHTAN, N. \& FREESTONE, M. 2015b. Psychiatric diagnosis and differential risks of offending following discharge. International Journal of Law and Psychiatry, 38, 68-74.

COID, J. W., YANG, M., ULLRICH, S., ZHANG, T., SIZMUR, S., FARRINGTON, D. \& ROGERS, R. 2011. Most items in structured risk assessment instruments do not predict violence. The Journal of Forensic Psychiatry and Psychology, 22, 3-21.

CONNELL, C. 2016. Forensic occupational therapy to reduce risk of reoffending: a survey of practice in the United Kingdom. The Journal of Forensic Psychiatry and Psychology, 27, 907-928.

CULLEN, A. E., CLARKE, A. Y., KUIPERS, E., HODGINS, S., DEAN, K. \& FAHY, T. 2012. A multisite randomized trial of a cognitive skills program for male mentally disordered offenders: violence and antisocial behavior outcomes. Journal of Consulting and Clinical Psychology, 80, 1114-20.

DISLEY, E., TAYLOR, C., KRUITHOF, K., WINPENNY, E., LIDDLE, M., SUTHERLAND, A., LILFORD, R., WRIGHT, S., MCATEER, L. \& FRANCIS, V. 2016. Evaluation of the offender liaison and diversion trials. Santa Monica, CA: RAND Corporation. www.rand.org/pubs/research_reports/RR1283.html.

DURCAN, G., HOARE, T. \& I., C. 2011. Pathways to unlocking secure mental health care. London, UK: Centre for Mental Health. 
EASTMAN, N., ADSHEAD, G., FOX, S., LATHAM, R. \& WHYTE, S. 2012a. Forensic Psychiatric Services. In: EASTMAN, N., ADSHEAD, G., FOX, S., LATHAM, R. \& WHYTE, S. (eds.) Oxford Specialist Handbooks on Forensic Psychiatry. Oxford, UK: Oxford University Press.

EASTMAN, N., ADSHEAD, G., FOX, S., LATHAM, R. \& WHYTE, S. 2012b. Legal Systems. In: EASTMAN, N., ADSHEAD, G., FOX, S., LATHAM, R. \& WHYTE, S. (eds.) Oxford Specialist Handbooks on Forensic Psychiatry. Oxford: Oxford University Press.

EASTMAN, N., ADSHEAD, G., FOX, S., LATHAM, R. \& WHYTE, S. 2012c. Treatment. In: EASTMAN, N., ADSHEAD, G., FOX, S., LATHAM, R. \& WHYTE, S. (eds.) Oxford Specialist Handbooks on Forensic Psychiatry. Oxford: Oxford university Press.

EDWORTHY, R., SAMPSON, S. \& VÖLLM, B. 2016. Inpatient forensic-psychiatric care: legal frameworks and service provision in three European countries. International Journal of Law and Psychiatry, 47, 18-27.

FAZEL, S., FIMINSKA, Z., COCKS, C. \& COID, J. 2016a. Patient outcomes following discharge from secure psychiatric hospitals: systematic review and meta-analysis. British Journal of Psychiatry, 208, 17-25.

FAZEL, S., GULATI, G., LINSELL, L., GEDDES, J. R. \& GRANN, M. 2009. Schizophrenia and violence: systematic review and meta-analysis. PLoS Med, 6, e1000120.

FAZEL, S., SINGH, J. P., DOLL, H. \& GRANN, M. 2012. Use of risk assessment instruments to predict violence and antisocial behaviour in 73 samples involving 24827 people: systematic review and meta-analysis. British Medical Journal, 345, e4692.

FAZEL, S., WOLF, A., FIMIŃSKA, Z. \& LARSSON, H. 2016b. Mortality, rehospitalisation and violent crime in forensic psychiatric patients discharged from hospital: rates and risk factors. PLOS ONE, 11, e0155906.

FAZEL, S., ZETTERQVIST, J., LARSSON, H., LANGSTROM, N. \& LICHTENSTEIN, P. 2014. Antipsychotics, mood stabilisers, and risk of violent crime. Lancet, 384, 1206-14.

FORRESTER, A., EXWORTHY, T., OLUMOROTI, O., SESSAY, M., PARROTT, J., SPENCER, S. J. \& WHYTE, S. 2013. Variations in prison mental health services in England and Wales. International Journal of Law and Psychiatry, 36, 326-32.

FROGLEY, C., TAYLOR, D., DICKENS, G. \& PICCHIONI, M. 2012. A systematic review of the evidence of clozapine's anti-aggressive effects. International Journal of Neuropsychopharmacology, 15, 1351-71.

GIBBON, S., DUGGAN, C., STOFFERS, J., HUBAND, N., VÖLLM, B. A., FERRITER, M. \& LIEB, K. 2010. Psychological interventions for antisocial personality disorder. Cochrane Database of Systematic Reviews, 16(6), Cd007668.

GOLENKOV, A., NIELSSEN, O. \& LARGE, M. 2014. Systematic review and meta-analysis of homicide recidivism and schizophrenia. BMC Psychiatry, 14, 46.

HARE DUKE, L., FURTADO, V., GUO, B. \& B., V. 2018. Long-stay in forensic-psychiatric care in the UK. Social Psychiatry and Psychiatric Epidemiology, 53, 313-321.

HOWNER, K., ANDINÉ, P., BERTILSSON, G., HULTCRANTZ, M., LINDSTRÖM, E., MOWAFI, F., SNELLMAN, A. \& HOFVANDER, B. 2018. Mapping systematic reviews on forensic psychiatric care: a systematic review identifying knowledge gaps. Frontiers in Psychiatry, 25, 452.

JCPMH 2013. Guidance for commissioners of forensic mental health services. Joint Commissioning Panel for Mental Health. www.jcpmh.info/resource/guidance-forcommissioners-of-forensic-mental-health-services/ 
KHIROYA, R., WEAVER, T. \& MADEN, T. 2009. Use and perceived utility of structured violence risk assessments in English medium secure forensic units. Psychiatric Bulletin, 33, 129-132.

LEUCHT, S., TARDY, M., KOMOSSA, K., HERES, S., KISSLING, W., SALANTI, G. \& DAVIS, J. M. 2012. Antipsychotic drugs versus placebo for relapse prevention in schizophrenia: a systematic review and meta-analysis. Lancet, 379, 2063-71.

LICHTENSTEIN, P., HALLDNER, L., ZETTERQVIST, J., SJOLANDER, A., SERLACHIUS, E., FAZEL, S., LANGSTROM, N. \& LARSSON, H. 2012. Medication for attention deficit-hyperactivity disorder and criminality. New England Journal of Medicine, 367, 2006-14.

MARTIN, M. S., DORKEN, S. K., WAMBOLDT, A. D. \& WOOTTEN, S. E. 2012. Stopping the revolving door: a meta-analysis on the effectiveness of interventions for criminally involved individuals with major mental disorders. Law and Human Behavior, 36, 112.

MEYNEN, G. \& OEI, K. 2011. Internationalizing forensic assessments of criminal responsibility. Medical Law Review, 30, 529-534.

MOLERO, Y., ZETTERQVIST, J., BINSWANGER, I. A., HELLNER, C., LARSSON, H. \& FAZEL, S. 2018. Medications for alcohol and opioid use disorders and risk of suicidal behavior, accidental overdoses, and crime. American Journal of Psychiatry, 175, 970-978.

NATARAJAN, M., SRINIVAS, J., BRISCOE, G. \& S., F. 2018. Community forensic psychiatry and the forensic mental health liaison model. Advances in Psychiatric Treatment, 18, 408415.

NEDOPIL, N. 2009. The role of forensic psychiatry in mental health systems in Europe. Criminal Behaviour and Mental Health, 19, 224-234.

NICE 2009. National Institute for Health and Care Excellence. Antisocial personality disorder: prevention and management [CG77]. London, UK: NICE. www.nice.org.uk/guidance/cg77

NJENGA, F. G. 2006. Forensic psychiatry: the African experience. World Psychiatry, 5, 97.

PERRY, A. E., NEILSON, M., MARTYN-ST JAMES, M., GLANVILLE, J. M., WOODHOUSE, R., GODFREY, C. \& HEWITT, C. 2015. Interventions for drug-using offenders with cooccurring mental illness. Cochrane Database of Systematic Reviews, 6, Cd010901.

SEPPÄNEN, A., TÖRMÄNEN, I., SHAW, C. \& KENNEDY, H. 2018. Modern forensic psychiatric hospital design: clinical, legal and structural aspects. International Journal of Mental Health Systems, 12, 1-12.

SIMPSON, A. I. 2015. Commentary: civil commitment and its reform. Journal of the American Academy of Psychiatry and the Law, 43, 48-51.

SINGH, J. P., GRANN, M. \& FAZEL, S. 2013. Authorship bias in violence risk assessment? A systematic review and meta-analysis. PLOS ONE, 8, e72484.

SINGH, J. P., SERPER, M., REINHARTH, J. \& FAZEL, S. 2011. Structured assessment of violence risk in schizophrenia and other psychiatric disorders: a systematic review of the validity, reliability, and item content of 10 available instruments. Schizophrenia Bulletin, 37, 899-912.

STEVENS, M. 2013. Broadmoor revealed: Victorian crime and the lunatic asylum. Barnsley, Yorkshire, UK: Pen and Sword Social History.

TOPIWALA, A. \& FAZEL, S. 2011. The pharmacological management of violence in schizophrenia: a structured review. Expert Review of Neurotherapeutics, 11, 53-63.

TROQUETE, N. A. C., VAN DEN BRINK, R. H. S., BEINTEMA, H., MULDER, T., VAN OS, T. W. D. P., SCHOEVERS, R. A. \& WIERSMA, D. 2013. Risk assessment and shared care 
planning in out-patient forensic psychiatry: cluster randomised controlled trial. British Journal of Psychiatry, 202, 365-371.

VÖLLM, B. A., EDWORTHY, R., HUBAND, N., TALBOT, E., MAJID, S., HOLLEY, J., FURTADO, V., WEAVER, T., MCDONALD, R. \& DUGGAN, C. 2018. Characteristics and pathways of long-stay patients in high and medium secure settings in England; a secondary publication from a large mixed-methods study. Frontiers in Psychiatry, 9, 140-140.

WHO 2018. Mental Health Atlas 2017. Geneva, Switzerland: World Health Organization. www.who.int/mental_health/evidence/atlas/mental_health_atlas_2017/en/

WILSON, H. A. 2014. Can antisocial personality disorder be treated? A meta-analysis examining the effectiveness of treatment in reducing recidivism for individuals diagnosed with ASPD. International Journal of Forensic Mental Health, 13, 36-46.

WILSON, S., JAMES, D. \& FORRESTER, A. 2011. The medium-secure project and criminal justice mental health. Lancet, 378, 110-111.

WITT, K., VAN DORN, R. \& FAZEL, S. 2013. Risk factors for violence in psychosis: systematic review and meta-regression analysis of 110 studies. PLoS One, 8, e55942.

WOLF, A., FANSHAWE, T. R., SARIASLAN, A., CORNISH, R., LARSSON, H. \& FAZEL, S. 2018. Prediction of violent crime on discharge from secure psychiatric hospitals: A clinical prediction rule (FoVOx). European Psychiatry, 47, 88-93.

WOLF, A., WHITING, D. \& FAZEL, S. 2017. Violence prevention in psychiatry: an umbrella review of interventions in general and forensic psychiatry. Journal of Forensic Psychiatry and Psychology, 28, 659-673.

ZHANG, S., MELLSOP, G., BRINK, J. \& WANG, X. 2015. Involuntary admission and treatment of patients with mental disorder. Neuroscience Bulletin, 31, 99-112. 